\title{
MBS taskforce fails the pub test
}

Follow this and additional works at: https://www.journal.acorn.org.au/jpn

Part of the Perioperative, Operating Room and Surgical Nursing Commons

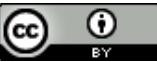

This work is licensed under a Creative Commons Attribution 4.0 License.

\section{Recommended Citation}

Hains, Toni; Smith, Catherine; and East, Rebecca (2021) "MBS taskforce fails the pub test," Journal of Perioperative Nursing: Vol. 34 : Iss. 1 , Article 4.

Available at: https://doi.org/10.26550/2209-1092.1116

https://www.journal.acorn.org.au/jpn/vol34/iss1/4

This Editorial is brought to you for free and open access by Journal of Perioperative Nursing. It has been accepted for inclusion in Journal of Perioperative Nursing by an authorized editor of Journal of Perioperative Nursing. 
Toni G Hains

PhD, MClinSc(PNSA), MNPractSt, NP, FACORN

\section{Catherine Smith}

MNPractSt, GradDipClinSc (PNSA), NP Australian College of Nurse

Practitioners

\section{Rebecca East}

MNursPrac(Perioperative),

MNursPrac(NursPrac), NP, FACORN Australian College of Perioperative Nursing

\section{MBS taskforce fails the pub test}

The nursing profession has been frustrated by a government review body that has ignored evidence-based recommendations resulting in a failure to meet community expectations. Politicians and the media have repeatedly labelled a failure to meet community expectations as failing the pub test.

Medicare, formerly Medibank and subsequently the Medicare Benefits Schedule (MBS), is funded by the federal government and was established in 1975 to improve health care access. The MBS operates by paying a 'fee for service' rebate to the patient for the cost of medical and allied health care in the private sector. Clinicians apply for a Medicare Provider Number, allowing the patient to access the rebate. To attract a rebate, services must be clinically relevant, which means the relevant health care professionals accept the service as necessary to provide appropriate treatment ${ }^{1}$, and listed on the MBS. Of the 5700 items listed on the MBS in 2010, nurse practitioners (NPs) were given access to four time-based consultation items². Including telehealth items introduced in 2011, NPs can now access a total of ten items. These items do not acknowledge the health care services NPs provide to Australian communities across various practice settings using expanded and advanced skills.

The Australian Government established the MBS Review Taskforce in 2015 to align the MBS with contemporary clinical evidence and practice and improve outcomes for patients ${ }^{3}$. The MBS Review Taskforce committee members were predominately medical practitioners who were tasked with reviewing the reports from all specialty reference groups and making recommendations to the federal health minister. The MBS Review Taskforce formed the Nurse Practitioner Reference Group (NPRG) in 2018 to evaluate existing MBS provisions and provide evidence-based recommendations to the MBS Review Taskforce related to NP services. The NPRG was comprised of NPS, medical practitioners and consumer representatives. The NPRG offered 14 evidence-based recommendations ${ }^{4}$ based on improving value for the patient and the health care system. These recommendations would enhance patient access to health care and were supported by peerreviewed evidence and real-world examples of patients' difficulties accessing care and NPs delivering care. Stakeholders, including the Australian College of Perioperative Nurses (ACORN), and consumer groups supported the NPRG recommendations due to the current MBS system leading to fragmented care, system inefficiencies, limited access and out-of-pocket expenses for health care consumers ${ }^{5}$.

None of these recommendations, which aligned with the MBS review's aims, were accepted by the MBS Review Taskforce. Of note is that the 14 recommendations by the NPRG were for funding of services already undertaken by NPS. The request for public funding by the MBS for these services was to support access to rebates for patients who have difficulty paying an out-of-pocket expense for the health care they already receive from an NP.

The taskforce did offer three alternative recommendations ${ }^{6}$, recommendations that clearly illustrate a lack of understanding of the NP's role and responsibilities 
by taskforce members?. The recommendations provided by the taskforce fall outside of the terms of reference of the MBS Review, were not supported by evidence and would impose an additional restriction on NPs' ability to provide what has been demonstrated to be safe, quality care $^{8}$.

The taskforce recommendation to endorse the continuation of mandatory collaborative agreements imposes medical oversight which is not a requirement for any other health care professional with access to the MBS. The 2010 legislation of the collaborative agreement was solely related to the funding of MBS rebates and access to medication subsidies for the Pharmaceutical Benefits Scheme for patients of NPS. The requirement for a collaborative agreement limits access to care for marginalised or underserved communities when medical practitioners, pathology services, imaging providers and pharmacists misinterpret the collaborative agreement's provisions leading to infringements on a legitimate NP scope of practice and, in some instances, breaching patient safety and privacy.

The MBS Review Taskforce request to establish a scope of practice and credentialing framework speaks to a lack of understanding and respect for the Nursing and Midwifery Board of Australia's (NMBA) robust regulatory frameworks for governance and endorsement of the NP. These processes are well established. It is a misconception that the NP scope of practice should be static. Like other health care professionals, the NP scope of practice must reflect continually developing best practice and meet the needs of the health care team and the patient ${ }^{9}$. It is the individual NP's responsibility to provide care that is within their level of competence, education and experience. Concerns regarding the scope of practice of any nurse are the responsibility of the NMBA, and this MBS Review Taskforce recommendation reflects the bias of the MBS review to the medico-centric health care system.

The final recommendation of the MBS Review Taskforce suggested an alternative funding pathway for NP services. It was a concern of the MBS Review Taskforce that funding NP services by endorsing the NPRG recommendations, would considerably expand the NP workforce with the potential that 'every nurse will become a nurse practitioner' . In reality, there is a worrying trend toward the Australian NP workforce's lack of growth compared to New Zealand?. The removal of NP funding barriers by the New Zealand government has resulted in a noticeable benefit to patients and communities. Rather than pursuing the costly and protracted process of creating alternative funding mechanisms for the NP, a federal focus on additional funding for all health care providers would support patient access to health care.

In the Year of the Nurse and the Midwife, and with Australia celebrating the $20^{\text {th }}$ anniversary of the NP, nursing as a profession has expressed extreme disappointment with the outcomes of the 2020 MBS Review Taskforce finding. Failure to give due consideration and endorse any of the NPRG's recommendations confirms the MBS Review Taskforce's limited understanding of Australians' health care needs and calls into question the credibility of the MBS review process.

The nursing profession, with 400000 voices, is the largest health care provider in the country. Individual nurses and nursing organisations must address the MBS review's failure to support not just NPs but nurses as a profession if nursing is to be viewed as the ally of the patient and not the enemy of the medical profession. ACORN was one of 40 nursing organisations to sign a rebuttal report, written by the Australian College of Nurse Practitioners in response to the MBS Review Taskforce's findings. This report is currently with the federal health minister.

As nurses, we must make our voices heard by lobbying federal members, participating in positive conversations with other health care professionals regarding the nursing profession's value, and participating in professional nursing organisations. Nurses deserve to be heard, and nurses must take their place at the health care policy table. We are the foundation on which the Australian health care system is built.

\section{References}

1. Biggs A. Medicare: A quick guide 2016 [Internet]. Canberra: Commonwealth of Australia; 2016 [cited 2021 February 4]. Available from: www.aph.gov.au/About_ Parliament/Parliamentary_Departments/ Parliamentary_Library/pubs/rp/rp1617/ Quick_Guides/Medicare.

2. Australian Government Department of Health. What legislation covers the MBS [Internet]. Canberra: Australian Government Department of Health; 2019 [cited 2021 February 4]. Available from: www.mbsonline.gov.au/internet/mbsonline/ publishing.nsf/Content/FAQ-Legislation.

3. Australian Government Department of Health. Medicare Benefits Schedule (MBS) Review [Internet]. Canberra: Australian Government Department of Health; 2019 [cited 2021 February 4]. Available from: www.health.gov.au/ internet/main/publishing.nsf/Content/ MBSReviewTaskforce. 
4. Nurse Practitioner Reference Group (NPRG). Report from the Nurse

Practitioner Reference Group to MBS Review Taskforce [Internet]. Canberra: Australian Government Department of Health; 2018 [cited 2021 February 4]. Available from: www1.health.gov.au/ internet/main/publishing.nsf/content/ BEB6C6D36DE56438CA258397000F4898/ \$File/NPRG\%20Final\%20Report\%20-\%20v2. pdf

5. Nurse Practitioner Reference Group (NPRG). Post Consultation Report from the Nurse Practitioner Reference Group [Internet]. Canberra: Australian Government Department of Health; 2019 [cited 2021 February 4]. Available from: www.health.gov.au/sites/default/files/ documents/2020/12/post-consultationreport-from-the-nurse-practitionerreference-group.pdf.

6. Medicare Benefits Schedule (MBS) Review Taskforce. Medicare Benefits Schedule (MBS) Review Taskforce Findings: Nurse Practitioner Reference Group Report [Internet]. Canberra: Australian Government Department of Health; 2020 [cited 2021 February 4]. Available from: www.health.gov.au/sites/default/files/ documents/2020/12/taskforce-findingsnurse-practitioner-reference-group-report pdf.

7. Australian College of Nurse Practitioners (ACNP). Response to MBS Taskforce Review - Report to The Honourable Greg Hunt MP [Internet]. Melbourne: ACNP: 2021 [cited 2021 February 4]. Available from: www.acnp.org.au/client_images/2242681.pdf.

8. White J, Thoms D, Bryant R, Chiarella M, Morton L. Medicare Benefits Schedule Taskforce Review. Letter to The Honourable Greg Hunt MP, 2020

9. Hains T, Smith C. What is the scope of practice of the nurse practitioner as a surgical assistant in Australia? JPN. 2020;33(3):e-16-e-21. DOI: doi. org/10.26550/2209-1092.1090. 